\title{
Multiple tumour emboli simulating a massive pulmonary embolus
}

\author{
J. W. HADFIELD* \\ M.B., M.R.C.P. \\ J. C. STERLING
M.A., B.Chir. \\ E. P. WRAIGHT \\ Ph.D., D.M.R.T. \\ Department of Respiratory Medicine, Papworth Hospital, Papworth Everard, Cambridgeshire, and
Department of Nuclear Medicine, Addenbrooke's Hospital, Cambridge
}

\section{Summary}

A 27-year-old man presented with shortness of breath due to multiple pulmonary emboli. At post-mortem the emboli consisted entirely of secondary epithelioid sarcoma, the primary having been resected 5 years previously.

\section{Introduction}

Pulmonary hypertension in association with carcinomatosis was first described over 100 years ago (Bristow, 1868). Kane et al. (1975), however, found only 23 reported cases of multiple tumour emboli causing dyspnoea, adding 16 of their own. Only four of these cases were reported from the British Isles. Sarcomas, although commonly metastasising to the lungs have not previously been reported as giving rise to multiple tumour emboli.

\section{Case report}

In September 1975 a 27 -year-old man presented with an ulcerating lesion on his right thumb. Biopsy showed it to be an epithelioid sarcoma. The tumour was excised with disarticulation of the thumb, the cut edge of the specimen being free from tumour histologically. The chest radiograph at this time was normal.

In October 1980 he developed gradually increasing dyspnoea. On admission to hospital 3 weeks later, he was found to be dyspnoeic at rest with reduced air entry to the left side of the chest and had a delayed pulmonary second sound. His chest radiograph showed loss of volume of the left lung and patchy pleural shadowing on the left side. The presumptive diagnosis was that the left lung was encased by secondary epithelioid sarcoma.

\footnotetext{
*Present address: Derbyshire Royal Infirmary, London Road, Derby.
}

On the day after admission he became more dyspnoeic and developed a pleural rub on the right side. Arterial blood gases showed a low $\mathrm{pO}_{2}$ and $\mathrm{pCO}_{2}$. An electrocardiogram showed right axis deviation and partial right bundle branch block. A ventilation-perfusion scan with ${ }^{133}$ xenon gas and ${ }^{99} \mathrm{~m}$ technetium labelled albumin micropheres (Fig. 1) showed absent perfusion in the left lung and multiple small peripheral defects in the right lung. Ventilation to the right lung was normal and reduced on the left corresponding to the radiologically reduced lung volume. He died 48 hours later.

\section{Post-mortem findings}

There were tumour nodules throughout the left parietal pleura and the left pleuro-pericardial membrane. Deposits of tumour were also found in the cerebellum, the pituitary stalk, the ileal mucosa and one small skin nodule in the left groin. Both lungs contained multiple areas of haemorrhagic consolidation but no macroscopic evidence of tumour or emboli.

On microscopy numerous medium and small sized pulmonary arteries were completely occluded by secondary sarcoma. It appeared that the emboli may have gained access to the circulation via a small epicardial vein which had been invaded by tumour.

\section{Discussion}

The most common malignancies associated with multiple tumour emboli are carcinoma of the breast, stomach, colon and cervix, and trophoblastic tumours, choriocarcinoma and hepatoma.

There are three reports of perfusion lung scans in patients with multiple tumour emboli. In two of these the appearances were of multiple peripheral segmental defects (Winterbauer, Elfenbein and Ball, 1968; 


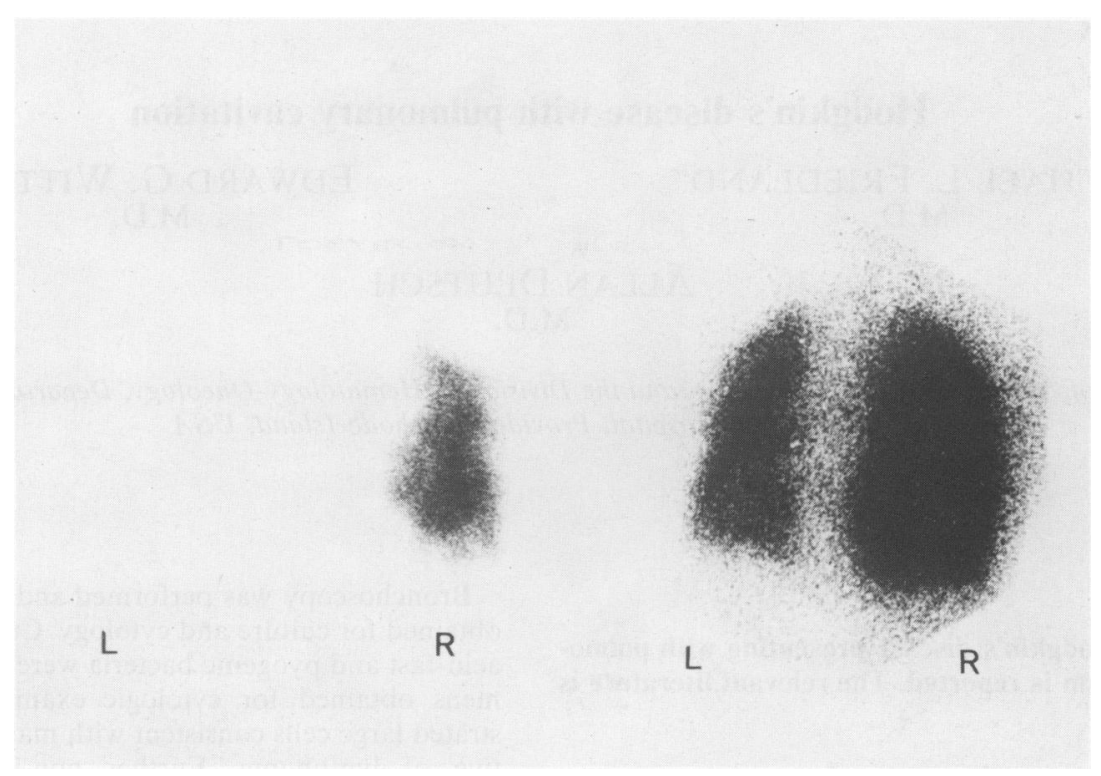

FIG. 1. Posterior view of perfusion scan (left) and ventilation scan (right).

Fanta and Compton, 1980) although in a third patient the scan was normal (case records, Massachusetts General Hospital, 1980). In our patient the absent perfusion to the left lung was interpreted as being due to a massive pulmonary embolus. However, perfusion scanning cannot differentiate between a massive pulmonary embolus and multiple micro-emboli affecting all the arterioles of one lung.

It is not possible to distinguish thrombotic embolism from tumour embolism in life without lung biopsy. However, with improvement in chemotherapy, the possibility of tumour emboli should be considered in patients with known malignancy and pulmonary emboli not responding to adequate anticoagulant therapy.

Epithelioid sarcoma is a rare tumour often mistaken for a chronic inflammatory process or a squamous cell carcinoma (Enzinger, 1970). The tumour occurs most frequently in young adults and affects the soft tissue of the hand, the distal forearm and the pre-tibial region. Epithelioid sarcomas are slow growing but recur after resection in $85 \%$ of cases. Late metastases occur in $30 \%$ and most commonly affect the lungs, particularly the pleura.

\section{Acknowledgments}

We would like to thank Dr J. E. Stark for permission to report this case, Dr P. G. I. Stovin for the post-mortem report and Miss Linda Fletcher for typing the manuscript.

\section{References}

BRISTOW, J.S. (1869) Colloid cancer of the oesophagus, stomach, lungs and adjoining lymphatic glands. Transactions of the Pathological Society of London, 19, 228.

Case Records of the Massachusetts General Hospital (case 43-1980) (1980) New England Journal of Medicine, 303, 1049.

ENZINGER, F.M. (1970) Epithelioid sarcoma. Cancer (Philadelphia), 26, 1029.

FANTA, C.H. \& CompTon, C.C. (1980) Microscopic tumour emboli to the lungs - a hidden cause of dyspnoea and pulmonary hypertension. Thorax, 35, 794.

Kane, R.D., Hawkins, H.K., Miller, J.A. \& Noce, P.S. (1975) Microscopic pulmonary tumour emboli associated with dysproea. Cancer (Philadelphia), 36, 1473.

Winterbauer, R.H., Elfenbein, I.B. \& Ball, W.C. (1968) Incidence and clinical significance of tumour embolisation to the lungs. American Journal of Medicine, 45, 271. 